\title{
DESIGN THINKING E POLÍTICAS PÚBLICAS
}

\author{
Lyvia Mendes Corrêa ${ }^{1}$ \\ Marcio Welter ${ }^{2}$ \\ João Bosco da Mota Alves ${ }^{3}$
}

\begin{abstract}
The participation of different social actors in the process of formulating and developing public policies has traditionally been reserved for political authorities. From this reality, it is questioned how design thinking contribute to the cycle of public policies? To answer this question, we conducted a systematic and integrative literature review, using the protocol proposed by Kitchenham (2004). We found that design thinking is applicable in the process of developing public policies, especially in the stages of preparing alternatives and making decisions, although with some criticisms, especially regarding scalability. Even so, it is a little explored topic that can be developed in future works, in addition to management and innovation processes, but also as a process for the elaboration of public policies, especially by the expression "policy design".
\end{abstract}

Keywords: design thinking; systematic review; policy design.

Resumo: A participação dos diversos atores sociais no processo de formulação e desenvolvimento das políticas públicas foi tradicionalmente reservada para autoridades políticas. A partir de tal realidade, questiona-se como o design thinking pode contribuir no ciclo de políticas públicas? Para responder tal pergunta, realizamos uma revisão sistemática e integrativa da literatura, utilizando o protocolo proposto por Kitchenham (2004). Verificamos que o design thinking é aplicável no processo de desenvolvimento de políticas públicas, principalmente nas fases de elaboração de alternativas e tomada de decisão, muito embora com algumas críticas, principalmente quanto à escalabilidade. Ainda assim, é um tema pouco explorado podendo ser desenvolvido em trabalhos futuros, para além de processos de gestão e inovação, mas também como processo de elaboração de políticas públicas, traduzida pela expressão policy design .

Palavras-chave: design thinking; revisão sistemática; elaboração de políticas públicas.

\footnotetext{
${ }^{1}$ Programa de Pós-Graduação em Engenharia e Gestão do Conhecimento - UFSC. Florianópolis - Brasil. Correio eletrônico: lyviacorrea@gmail.com.br

${ }^{2}$ Programa de Pós-Graduação em Engenharia e Gestão do Conhecimento - UFSC. Florianópolis - Brasil. Correio eletrônico: contato@marciowelter.com.br

3 Programa de Pós-Graduação em Engenharia e Gestão do Conhecimento - UFSC. Florianópolis - Brasil. Correio eletrônico: jbosco@inf.ufsc.br
} 


\section{INTRODUÇÃO}

A política pública é o campo do conhecimento que busca ao mesmo tempo colocar o governo em ação bem como analisar essa ação, tida como a variável independente e, quando necessário, propor mudanças no rumo ou curso dessas ações: variável dependente. Uma boa política pública deve aproveitar o ponto de vista entre as pessoas que irão ser impactadas pelas propostas (Souza, 2006; Mintrom \& Luetjens, 2016).

Conforme Clarke \& Craft (2019) a formulação de políticas públicas pode apresentar algumas limitações como: a) o contexto político no qual o projeto de políticas pública ocorre e a aplicação restrita para políticas que focam no usuário, seguindo a experiência da iniciativa privada; b) recursos humanos, financeiros e organizacionais necessários; c) diferentes estilos políticos e arranjos governamentais que podem ser apropriados para cada política setorial, jurisdições e problemas políticos; d) a necessidade de que os projetos tenham interoperabilidade em diferentes mixes de políticas, com outros instrumentos e objetivos.

Neste âmbito, o Design Thinking formulado por Brown (2008) surge como um processo alternativo para a geração do conhecimento: auxiliando na definição do problema e na buscar a solução, além de enfatizar a co-criação, considerando as visões e recursos de uma ampla gama de atores sociais. Dessa forma, aumenta-se a possibilidade de as políticas públicas terem os efeitos pretendidos. (Clarke \& Craft, 2019; Mintrom \& Luetjens, 2016).

Por outro lado, para considerar o potencial da utilização de design thinking nas fases do ciclo de políticas públicas, deve-se aprofundar a análise, objetivo e contribuição central desta revisão. Inicialmente apresentaremos uma conceituação acerca de design thinking e de política pública, temas centrais deste artigo, em seguida apresentaremos os procedimentos metodológicos utilizados, passaremos à análise e discussão dos resultados encontrados e, por fim, apresentaremos considerações finais sobre nosso estudo.

\section{REFERENCIAL TEÓRICO}

Souza (2006) lembra que, para se transformarem em políticas públicas, problemas públicos precisam encontrar o equilíbrio entre o que é tecnicamente eficiente e também o que é politicamente viável.

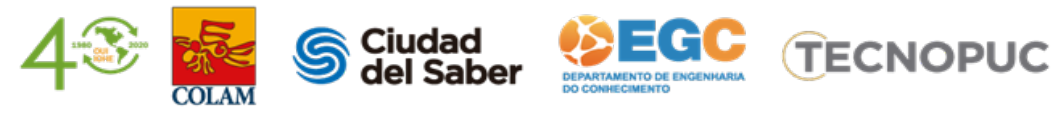


O design thinking promete suprir a lacuna comum na administração pública entre os objetivos da formulação de políticas e as experiências dos cidadãos ao interagirem com os serviços do governo, sem a presunção de partir de uma resposta conhecida ou mesmo de um problema bem definido (Mintrom \& Luetjens, 2016).

Nesta seção faremos uma breve fundamentação teórica sobre o que é o design thinking e o que entendemos por políticas públicas.

\subsection{DESIGN THINKING}

Para Tim Brown (2008) o processo de design consiste em um sistema de espaços, diferente do que tradicionalmente ocorre em um processo com uma série predefinida de etapas ordenadas. Afirma que os espaços demarcam diferentes tipos de atividades relacionadas, porém, juntas formam o continuum da inovação. $\mathrm{O}$ autor salienta que este processo de design pode parecer caótico ao ser experimentado pela primeira vez, entretanto, durante a execução do projeto o processo faz sentido e alcança resultados, mesmo tendo uma arquitetura diferente dos processos lineares baseados em marcos típicos de outros tipos de atividades.

Os projetos de design devem passar por três espaços: Inspiração, Ideação e Implementação. Brown (2008) define que "Inspiração" é o espaço onde as circunstâncias (sejam elas um problema, uma oportunidade ou ambos) motivam a busca de soluções; "Ideação", para o processo de geração, desenvolvimento e teste de ideias que podem levar a soluções; e "Implementação", para o mapeamento de um caminho para o mercado, ou seja a aplicação sobre a circunstância problema. Por fim, reafirma que os projetos voltarão a percorrer esses espaços - especialmente os dois primeiros - mais de uma vez, à medida que surgem novas ideias ou quando refinadas.

Os métodos de solução de problemas que sustentam as ciências políticas demandam uma mentalidade analítica e lógica do pensador para que divida fenômenos complexos em partes gerenciáveis, que podem ser avaliadas racionalmente, logicamente e através do raciocínio dedutivo (Clarke \& Craft, 2019). Essa abordagem baseia-se no ato de "pensar as coisas através” e geralmente depende de uma lente única ou limitada. (Bason, 2010)

Em contraste, conforme salientam Clarke e Craft (2019), o estilo de pensamento interpretativo-intuitivo incluído no design thinking também prioriza a síntese, reunindo o

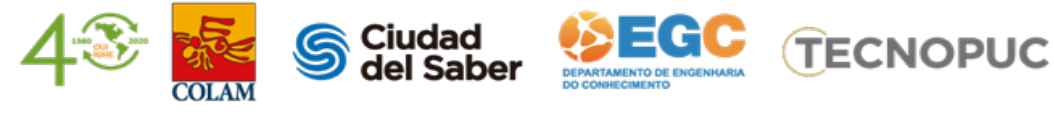


fenômeno complexo e avaliando-o como um todo, conduzindo o pensador a adotar visões de problemas baseadas em sistemas e desenhando em uma lente interdisciplinar e com múltiplas perspectivas, o qual pode contribuir para as políticas públicas, conforme trataremos a seguir.

\subsection{POLÍTICAS PÚBLICAS}

Howlett (2014) caracteriza política pública como o resultado dos esforços realizados pelos governos para alterar aspectos próprios ou do comportamento social com o objetivo de atingir um objetivo ou propósito, sendo esses mais ou menos projetados.

Também se pode entender a política pública como uma diretriz elaborada para enfrentar um problema público a qual possui dois elementos fundamentais, quais sejam: a intencionalidade pública e resposta a um problema público. (Secchi, 2014)

A formulação de políticas foi considerada pelos policy makers com um caminho linear desde a definição do problema até a avaliação das opções (Mintron; Luetjens 2016), entretanto, neste trabalho, para relacionarmos o design thinking com o processo de desenvolvimento de políticas públicas, partiremos da proposição de Lindblom (1959), que defendeu que elaboração de políticas deve ser feita e refeita infinitamente, como em um ciclo.

Figura 2. Ciclo de Políticas Públicas

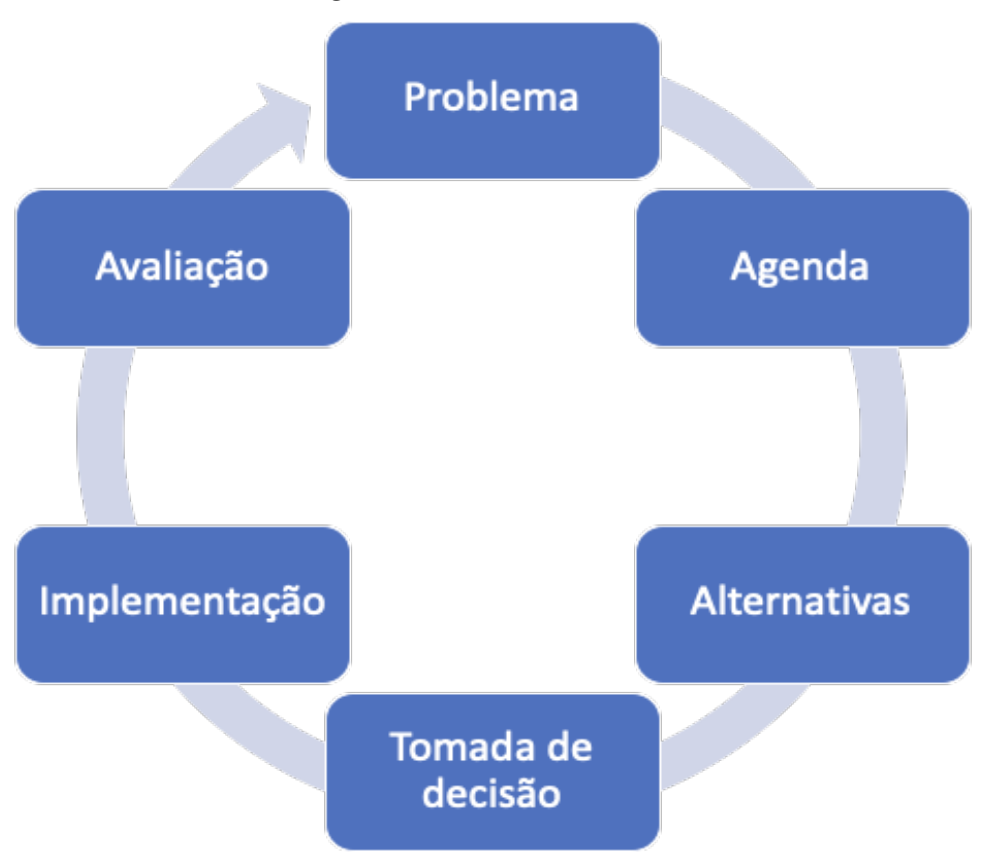

Fonte: Autores (2020) a partir de Lindblom (1959)

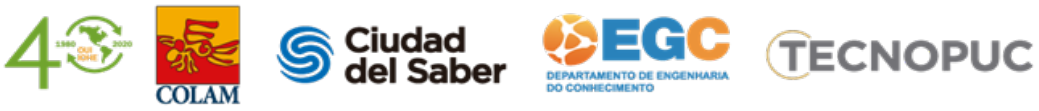


Para compreensão das fases do ciclo, temos que o problema reflete uma discrepância entre o status quo e uma situação ideal possível. Um problema público é a diferença entre o que é, e aquilo que se gostaria que fosse a realidade pública (Secchi, 2014). É a definição do problema que dará origem à agenda, que consiste conjunto de assuntos sobre os quais o governo e pessoas ligadas a ele concentram sua atenção num determinado momento (Kingdom, 2003).

Estando o problema constante da agenda, passa-se a gerar alternativas: momento em que são elaborados métodos, programas, estratégias ou ações que poderão alcançar os objetivos estabelecidos, sendo que um mesmo objetivo pode ser alcançado de várias formas, por diversos caminhos. A fase seguinte é a tomada de decisão, que trata do momento em que os interesses dos atores são equacionados e as intenções (objetivos e métodos) de enfrentamento de um problema público são demonstradas (Secchi, 2004).

A implementação é caracterizada pela maior liberdade de burocratas e redes de atores em auto organizar e modelar a implementação de políticas públicas e a avaliação, envolve a medição do grau em que vários grupos de atores e o público interessados valorizam os resultados ou atributos possíveis do programa ou política (Secchi, 2004; Mark, Henry \& Julnes, 1999).

A visão do processo de desenvolvimento das políticas como um ciclo demonstra que uma política pública pode não necessariamente chegar a um fim, sendo seu desenho retroalimentado por alterações no cenário, nos objetivos e pela atuação dos atores envolvidos.

\section{PROCEDIMENTOS METODOLÓGICOS}

Como suporte na revisão da literatura optou-se pelo protocolo de revisão proposto por Kitchenham (2004) e inspirado pelos trabalhos de aplicabilidade de Kitchenham e Charters (2007). Constam do protocolo da pesquisa: necessidades e escopo da pesquisa, estratégia de busca dos artigos, os critérios de seleção e exclusão, dados extraídos e limitações e validações do trabalho. 


\subsection{QUESTÃO DE PESQUISA}

Como o design thinking pode contribuir na geração de conhecimento ao longo do ciclo de políticas públicas?

\subsection{ESTRATÉGIA DE BUSCA}

Esta revisão buscou pelos descritores design thinking, public policy, public policies, policymaking, policy-making, policy making, citizen, citizens, government, national policy, política pública, políticas públicas, cidadão, cidadãos, política nacional, política do estado, política de estado, plano nacional, plano de governo, plano estadual, plano municipal, governo, policy design nas bases da Scopus, ProQuest, WebOfScience, SocINDEX EBSCOhost, Scielo WebOfScience, BDTD - Biblioteca Digital Brasileira de Teses e Dissertações e Catálogo de Teses e Dissertações CAPES.

Tais descritores foram determinados a partir da contextualização de Secchi (2014) o qual destaca a diferença entre politics e policy, já que nos países de matriz linguística latina os dois termos possuem correspondência à uma única tradução: "política". Também foram utilizadas variações de termos em inglês, restringindo a tipos de política em português, para direcionar a efetividade da pesquisa em meio a pluralidade semântica do verbete "política", de tal modo que ampliasse as possibilidades de encontrar trabalhos relevantes sobre design thinking e políticas públicas.

Assim, a partir dessas considerações, combinamos os descritores por meio de operadores booleanos, formando os seguintes extratos de busca: ( "design thinking") AND ( “public policy” OR “public policies” OR policymaking OR policy-making OR “policy making” OR citizen OR citizens OR government OR "national policy" OR "política pública” OR “políticas públicas” OR cidadão OR cidadãos OR "política nacional” OR “política do estado" OR “política de estado” OR “plano nacional” OR "plano de governo” OR “plano estadual” OR “plano municipal” OR governo OR “policy design” 


\subsection{CRITÉRIOS DE INCLUSÃO/EXCLUSÃO}

Os critérios de inclusão e exclusão adotados foram explicitados no quadro 1, que segue:

Quadro 1 - Critérios de Seleção

\begin{tabular}{|l|c|}
\hline \multicolumn{2}{|c|}{ Critério de exclusão } \\
\hline E1 & Ausência das palavras-chave no título e nem no resumo \\
\hline E2 & Brown (2008) \\
\hline E3 & Não aborda temas de abrangência das políticas públicas de Governo \\
\hline E4 & vinculação explícita enquanto proveniente ou para concepção de política pública no setor público sem \\
\hline E5 & Idioma diferente de Inglês, Espanhol ou Português \\
\hline I1 & \\
\hline I2 & Apresenta elementos sobre política e demandas sociais \\
\hline
\end{tabular}

Fonte: Autores (2020)

\subsection{SELEÇÃO DOS ARTIGOS}

A quantidade de trabalhos encontrados nas bases por meio da estratégia de busca planejada e após a aplicação dos critérios de seleção está retratada na Tabela 1.

Tabela 1. Quantidade de trabalhos encontrados por base de dados

\begin{tabular}{|l|c|c|}
\hline \multicolumn{1}{|c|}{ Base de dados } & Trabalhos encontrados & Selecionados \\
\hline Scopus & 127 & 1 \\
\hline ProQuest & 17 & - \\
\hline WebOfScience & 80 & 5 \\
\hline
\end{tabular}




\begin{tabular}{|l|c|c|}
\hline SocINDEX EBSCOhost & 2 & - \\
\hline Scielo WebOfScience & 1 & - \\
\hline BDTD & 9 & - \\
\hline Catálogo de Teses e Dissertações CAPES & 222 & $\mathbf{6}$ \\
\hline Total & $\mathbf{4 5 8}$ & - \\
\hline
\end{tabular}

Fonte: Autores (2020)

O fluxo de refinamento do resultado das buscas se deu conforme segue:

Tabela 2. Fluxo de seleção de documentos encontrados nas bases de dados

\begin{tabular}{|c|c|}
\hline Documentos Encontrados & 458 \\
\hline Duplicados & 47 \\
\hline Documento não era trabalho científico & 6 \\
\hline Não atendeu ao critério de idioma & 6 \\
\hline Eliminado por título, resumo e palavras-chave & 360 \\
\hline Eliminados após leitura integral & 33 \\
\hline Selecionados & $\mathbf{6}$ \\
\hline
\end{tabular}

Fonte: Autores (2020)

\subsection{EXTRAÇÃO DOS DADOS}

Para melhor analisar os artigos selecionados pelo critério de inclusão deste mapeamento sistemático, foram extraídos dos artigos os seguintes dados:

Quadro 2. Atributos da extração de dados

\begin{tabular}{|l|l|}
\hline id & identificador numérico sequencial. \\
\hline situação & $\begin{array}{l}\text { identificação da seleção do artigo, importando apenas os resultantes em } \\
\text { "selecionado". }\end{array}$ \\
\hline base de dados & nome da base de dados que o artigo foi encontrado. \\
\hline data da consulta & data e hora em que foi realizada a busca na base de dados. \\
\hline
\end{tabular}




\begin{tabular}{|l|l|}
\hline Abordagem & ênfase temática do artigo quanto às fases do ciclo de política pública. \\
\hline título & título do artigo \\
\hline autores & nomes dos autores do artigo \\
\hline ano & ano da publicação do artigo \\
\hline resumo & resumo do artigo na língua original \\
\hline palavras-chave & palavras-chaves em português \\
\hline keywords & palavras-chave em inglês \\
\hline idioma & referência ao idioma do conteúdo integral do artigo \\
\hline URL ou DOI & endereço eletrônico ao artigo, detalhe ou DOI \\
\hline
\end{tabular}

Fonte: Autores (2020)

Como suporte para a classificação e critério de qualidade dos trabalhos além de atenderem aos critérios de exclusão e inclusão previamente definidos, deveriam minimamente passar por revisão por pares, demonstrar a aplicabilidade dos métodos e apresentar resultados no âmbito pesquisado. A falta de evidências da validação dos trabalhos encontrados ou trabalhos apresentando apenas suposições ou conceitos foram descartados.

\section{RESULTADOS}

Conforme demonstrado na tabela 1, ao final da seleção, restaram seis artigos, sendo um da base de dados Scopus e cinco da base de dados WebOfScience, publicados entre os anos de 2012 e 2019. Na base de dados Scopus encontramos Chindarkar, Howlett \& Ramesh (2017) e a base WebOfScience retornou os artigos de Clark \& Craft (2019), Mcgann, Blomkamp \& Lewis (2018), Mintron \& Luetjens (2016), Lee, Ma \& Zhou (2016) e Hobday, Boddington \& Grantham (2012) o qual apresentou o maior número de citações.

Categorizamos os trabalhos com base na relação estabelecida entre a fase do ciclo de desenvolvimento da política pública e a possibilidade de aplicação do design thinking, conforme a figura 4. 
Figura 4. Relação entre os trabalhos e adoção do DT nas fases do ciclo de políticas públicas
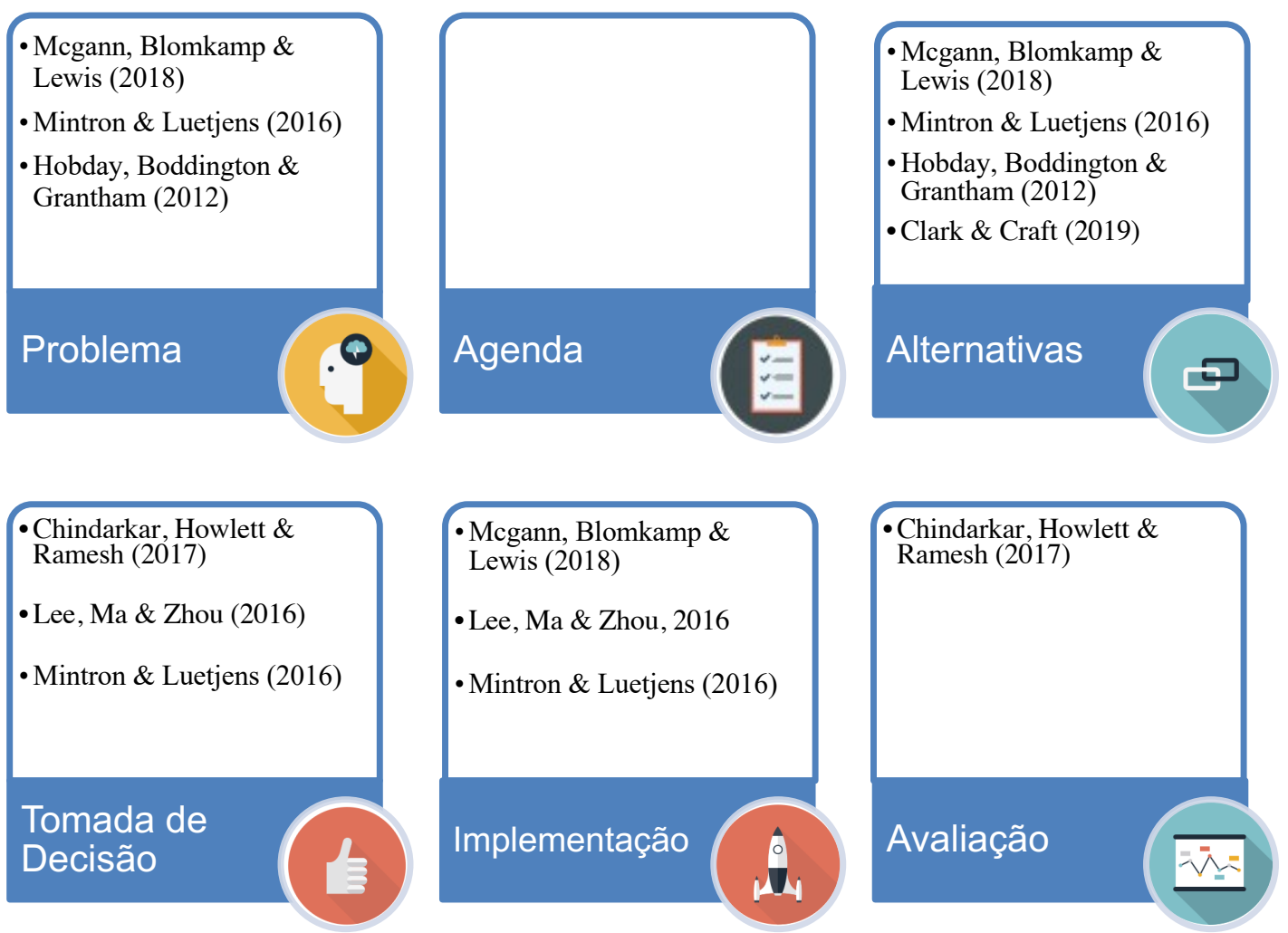

Fonte: Autores (2020)

A participação dos diversos atores sociais no processo de formulação e desenvolvimento das políticas públicas foi tradicionalmente reservada para ao governo e somente nos últimos anos estudos acadêmicos investigaram a participação de atores interessados, ainda que haja dúvidas sobre quais as metodologias usadas (Pluchinotta et al., 2019), o que pode explicar o número reduzido de trabalhos encontrados especificamente dentro da proposta deste trabalho.

Ademais, da análise dos resultados da busca, verifica-se que a maior parte dos trabalhos tem como enfoque a utilização do design thinking nas fases de definição do problema e geração de alternativas, não tendo sido encontrados artigos que tratam de forma específica sobre a construção da agenda e sua relação com o processo de design thinking.

Isso pode ser explicado, dado que a maior parte das abordagens de participação dos diversos atores sociais no desenvolvimento de políticas públicas serve para atrair os interessados para o processo de decidir entre opções, em como escolher entre alternativas, mas não em considerar como isso pode ser estabelecido (Pluchinotta et al., 2019). 


\section{DISCUSSÃO}

Os formuladores de políticas muitas vezes aceitam, por padrão, a visão racional da política como uma como atividade programável e passível de intervenções sistemáticas, planos, metas e correções, mas o design thinking procura abordar o caráter social e criativo da política, incorporando o lado humano do processo de design, reinterpretando a formulação de políticas como uma atividade social com ciclos de feedback, propriedades emergentes e consequências não intencionais (Hobday, Boddington \& Grantham, 2012).

Os autores relacionaram a noção de wicked problems vinda do design thinking à definição de "problemas complexos" de Lindblom (1959) o qual defendeu que, sob condições de incerteza e informações incompletas, um passo-a-passo é a maneira mais racional de proceder. Na visão dos autores, um problema complexo requer um processo criativo e social para gerar soluções e seguir com um programa flexível programa de mudanças

Mintron e Luetjens (2016) aduzem que no desenvolvimento de uma política pública, os policy makers costumam efetuar a consulta às partes interessadas apenas no final do processo, depois que a definição do problema ocorreu, as opções foram analisadas e os caminhos amplamente aceitáveis foram explorados.

Acreditam que a consulta em um estágio posterior reduz o risco de o trabalho político ser submetido a um grande desafio e ter de ser refeito, outrossim, há o risco de a consulta ser considerada uma mera formalidade, com o objetivo de limitar a capacidade das partes interessadas seriamente serem informadas sobre o mecanismo de desenvolvimento da política (Mintron e Luetjens, 2016).

Nesse sentido, sustentam a importância do engajamento inicial das partes interessadas, destacando a importância do design thinking como um caminho para a definição do problema a ideação de alternativas e a tomada de decisão, destacando que o gestor deve trabalhar para garantir que todos os envolvidos entendam por que estão sendo consultados e como a consulta está sendo sequenciada (Mintron \& Luetjens, 2016).

Diferente dos demais trabalhos discutidos neste artigo, Chindarkar, Howlett e Ramesh (2017) discorrem sobre a possibilidade de uso do design thinking não a partir de uma perspectiva centrada no usuário e sim, centrada no problema. Assim, a ênfase está na

\section{TECNOPUC}


participação dos diversos atores sociais nos processos de tomada de decisão e de avaliação das políticas públicas.

Os autores esperam que cada vez mais os governos colocarão em prática os recursos do design thinking e utilizarão as evidências do próprio governo, do público e dos especialistas, especialmente onde os cidadãos têm participação direta, para a formulação de políticas. (Chindarkar, Howlett \& Ramesh, 2017)

Lee, Ma e Zhou (2016) narram a transição ocorrida no governo da cidade-estado de Cingapura, na Malásia, desde o início da autonomia, em 1959 quando as políticas públicas eram marcadas por uma abordagem pragmática e "de cima para baixo", até os dias de hoje, com participação e colaboração dos atores sociais no desenho e implementação de políticas.

Neste sentido, o design thinking vem sendo usado para possibilitar a ampla participação da sociedade, focando em alcançar resultados sustentáveis e valor público, em vez de abordar soluções rápidas. O uso da ideação e prototipação no âmbito dos laboratórios de inovação governamentais também possuem papel de importância.

A atuação dos laboratórios difere da atuação da formulação tradicional de políticas públicas na medida em que envolvem significativamente os atores não-tradicionais da política nesse processo, a aplicação do design thinking convida uma gama mais diversa de vozes e insumos no processo político que ressoa com princípios de governança de rede (Mcgann, Blomkamp \& Lewis, 2018).

Os PSI Labs (sigla em inglês para Public Setor Innovation Laboratories) enfatizam a aplicação do design thinking à política e priorizam métodos "centrados no usuário" como etnografia, técnicas de visualização e colaboração com cidadãos e outras partes interessadas para esclarecer definições de problemas e co-criar soluções (Mcgann, Blomkamp \& Lewis, 2018).

A importância de abordagens de design thinking está em desafiar a formulação de políticas públicas tradicionais e a tomada de decisões, pois requer habilidades específicas como etnografia, ciências comportamentais, comunicação e arquitetura. Também requer a quebra de silos organizacionais e processuais, contestando hierarquias e burocracias. Portanto, laboratórios de inovação ou design são estabelecidos com afiliação direta ou indireta a governos para servir como catalisadores para o design thinking (Lee, Ma \& Zhou, 2016). 
Lee, Ma e Zhou (2016) sintetizam que as abordagens participativas como o design thinking sustentam um conceito de governança colaborativa que abrange a contribuição do governo, mercado e sociedade para produzir maior valor público a um custo menor.

\subsection{CRÍTICAS À ADOÇÃO DO DESIGN THINKING NO DESENVOLVIMENTO DE POLÍTICAS PÚBLICAS}

Apesar de sua popularidade global, tem havido pouca avaliação crítica do design thinking a partir de uma perspectiva de governança pública, sobretudo por muitas aplicações serem praticamente desprovidas das considerações metodológicas, analíticas, empíricas e normativas da literatura de desenvolvimento de políticas públicas (Clarke \& Craft, 2017).

Há de se considerar que, se o design thinking é um sistema que não está em conformidade com a visão racional de tomada de decisão, ferramentas de elaboração de políticas podem ser ineficazes ou mesmo contra produtivas (Hobday, Boddington \& Grantham, 2012).

Muito embora sejam justificadas as críticas dos defensores do design thinking acerca das formas tradicionais de formulação de políticas por usualmente não considerar as experiências vividas do usuário afetado pelo problema e o impacto real dos projetos de políticas criados para abordar esses problemas, o centrismo do usuário não é um princípio de design facilmente aplicável ou mesmo apropriado para todas os contextos. (Clarke \& Craft, 2017). Os mesmos autores enumeram outras críticas aplicação do design thinking no desenvolvimento de políticas públicas.

Primeiramente destacam que, ao se considerar a formulação de políticas que são politicamente controversas (a exemplo de problemas como combate a violência, enfrentamento a questões relacionadas ao uso e comercialização de drogas), ficaria evidenciada a primeira falha do design thinking, que é ignorar a política envolvida no processo político (Clarke \& Craft, 2017).

Uma segunda deficiência encontrada no design thinking surgiria quando se considera a escalabilidade: o processo de design thinking pode não ser apropriado para todos os contextos de políticas, dado o sequenciamento, os horizontes de tempo e a intensidade de recursos que ele exige. Alguns problemas de política exigem decisões imediatas e muitas vezes os recursos 
necessários para testes do usuário, iteração e colaboração interna e externa não estão disponíveis. (Clarke \& Craft, 2017).

Clarke e Craft (2017) ainda argumentam que o design thinking tende a privilegiar os arranjos de governança em rede, o que, segundo os autores, é simplista demais, já que existem muitos outros estilos de políticas e arranjos de governança relacionados que podem ser adequados a um dado problema de política. Também ignora que os projetos de políticas são aninhados e combinados com uma variedade de outros projetos de políticas com os quais eles interagem.

Em relação as críticas, Mintron e Luetjens (2016) ponderam que a eficácia do design thinking dependerá da compreensão e intenção dos usuários. É um processo demorado e não deve ser realizado para ganhos de eficiência e para que a mudança ocorra, o design thinking requer liderança e comprometimento. Também reconhecem que "esforços são necessários para determinar as condições sob as quais o design thinking melhora seriamente a formulação de políticas".

\section{CONCLUSÃO}

Ao considerarmos que os problemas complexos a serem enfrentados pelas políticas públicas podem ser comparados aos wicked problems, a formulação de políticas requer um processo criativo e social para gerar soluções e seguir com um programa flexível programa de mudanças, como o design thinking (Hobday, Boddington \& Grantham, 2012).

Atualmente, o estudo da aplicação do design thinking em políticas públicas vem sendo desenvolvido, em sua maioria, no âmbito dos laboratórios de inovação no setor público, que contribuem como parte das modernas organizações públicas A identificação das ações destes laboratórios na realidade serve como exemplo do potencial da utilização do design thinking para as diversas fases das políticas públicas (Mcgann, Blomkamp \& Lewis, 2018).

Entretanto, críticos do design thinking no desenvolvimento de políticas públicas aduzem que tal processo não serve para substituir integralmente as atuais metodologias de desenvolvimento de políticas públicas (Clarke \& Craft, 2017)

Por outro lado, uma nova forma de pensar políticas públicas requer aprendizado organizacional e mudança cultural. Portanto não se pode esperar que haja uma mudança para 
um paradigma diferente, sem a visão, apoio e compromisso de longo termo dos líderes políticos. (Head, 2008)

Esta abordagem complementa a visão dos sistemas de inovação de um complexo mundo com organizações incorporadas em um sistema diversificado e dinâmico. Um reequilíbrio a favor da formulação de políticas centradas no ser humano pode potencialmente ser uma nova arena útil para a pesquisa de políticas (Hobday, Boddington \& Grantham, 2012).

Por fim, temos que o uso do design thinking no ciclo de políticas públicas é um tema pouco explorado e ainda em desenvolvimento para trabalhos futuros, para além de processos de gestão ou de inovação, mas também como processo de elaboração de políticas públicas, traduzida pela expressão policy design e, especialmente, nos campos das Ciências Sociais e Política, a exemplo nos trabalhos de Howlett, Ramesh e Perl (2009) e Howlett (2014).

\section{REFERÊNCIAS}

Bason, C. (2010). Leading Public Sector Innovation. Co-Creating for a Better Society. Bristol University: Policy Press.

Brown, T. et al. (2008) Design thinking. Brighton: Harvard Business Review, 86 (6).

Chindarkar, N., Howlett, M., \& Ramesh, M. (2017). Introduction to the Special Issue: "Conceptualizing Effective Social Policy Design: Design Spaces and Capacity Challenges." Public Administration and Development, 37(1), 3-14. https://doi.org/10.1002/pad.1789.

Clarke, A., \& Craft, J. (2019). The twin faces of public sector design. Governance, 32(1), 521. https://doi.org/10.1111/gove.12342.

Head, B. (2008) Wicked Problems in Public Policy. Public Policy. [s.1.], 3 (2), 101-108. Curtin University of Tecnology.

Hobday, M., Boddington, A., \& Grantham, A. (2012). Policies for design and policies for innovation: Contrasting perspectives and remaining challenges. Technovation, 32(5), 272-281. https://doi.org/10.1016/j.technovation.2011.12.002

Howlett, M, Perl, A., \& Ramesh, M. (2009). Studying Public Policy: Policy Cycles \& Policy Subsystems. Oxford University Press. Retrieved from https://books.google.com.br/books?id=9I1zPwAACAAJ

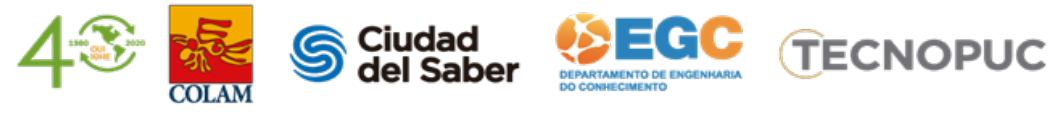


Howlett, Michael. (2014). From the "old" to the "new" policy design: Design thinking beyond markets and collaborative governance. Policy Sciences, 47(3), 187-207. https://doi.org/10.1007/s11077-014-9199-0.

Kitchenham, B. (2004). Procedures for performing systematic reviews, Joint Technical Report Software Engineering Group, Department of Computer Science, Keele University, United King and Empirical Software Engineering, National ICT Australia Ltd., Australia.

Kitchenham, B; Charters, S. (2007). Guidelines for performing Systematic Literature Reviews in Software Engineering. Evidence-Based Software Engineering (EBSE). Keele: KeeleUniversity; Durham: Universityof Durham.

Lee, C., Ma, L., \& Zhou, Y. (2017, September 2). The changing dynamics of policy experiment in Singapore: does the 2011 general election make a difference? Asian Journal of Political Science. Routledge. https://doi.org/10.1080/02185377.2017.1351382.

Lindblom, C. E. (1959) The Science of Mudding Though. Public Administration Review. 19 (2): 79-88.

Mark, M. M.; Henry, Gary T.; Julnes, George. (1999) Toward an Integrative Framework for Evaluation Practice. American Journal of Evaluation, 20 (2):177-198. SAGE Publications. http://dx.doi.org/10.1177/109821409902000202.

McGann, M., Blomkamp, E., \& Lewis, J. M. (2018). The rise of public sector innovation labs: experiments in design thinking for policy. Policy Sciences, 51(3), 249-267. https://doi.org/10.1007/s11077-018-9315-7.

Mintrom, M., \& Luetjens, J. (2016). Design Thinking in Policymaking Processes: Opportunities and Challenges. Australian Journal of Public Administration, 75(3), 391-402. https://doi.org/10.1111/1467-8500.12211

Mulgan, G. (2014). Design in public and social innovation: what works and what could work better? London, UK: Nesta.

Pluchinotta, I. et al. (2019) Design Theory for Generating Alternatives in Public Decision Making Processes. Group Decision And Negotiation, 28(2), 41-375. Springer Nature. http://dx.doi.org/10.1007/s10726-018-09610-5

Secchi, L. (2014) Políticas Públicas: conceitos, esquemas de análise, casos práticos. São Paulo: Cengage Learning. 